\title{
Vladimir Voevodsky - Work and Destiny
}

Mark Bickford (Cornell University, Ithaca, USA), Fedor Bogomolov (New York University, USA) and Yuri Tschinkel (New York University and Simons Foundation, New York, USA)

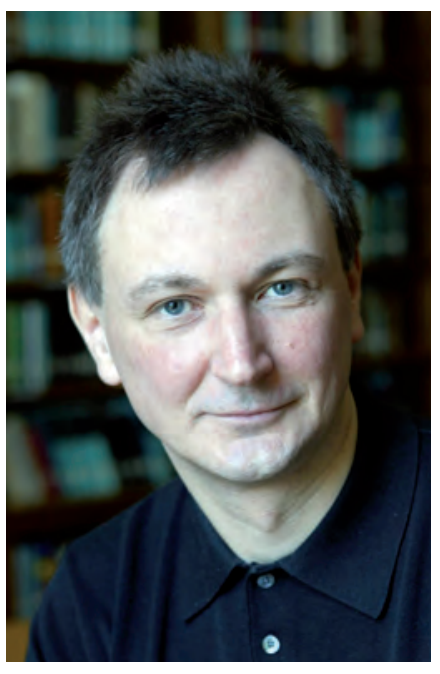

Vladimir Voevodsky, who died in Princeton on 30 September 2017 at the age of 51 , was one of the most remarkable and highly original mathematicians of our time. His achievements have been recognised with the highest honour of the profession, the Fields Medal, which he received in 2002. His work transformed several fields of mathematics and theoretical computer science.

Vladimir started his mathematical education as a high school student attending the Shafarevich seminar at the Steklov Institute of the Russian Academy of Sciences in Moscow. He bypassed the "usual" mathematical Olympiad training and moved directly into research. His exceptional talent and focus were already apparent then to all who interacted with him. As an undergraduate student at Moscow State University, he fully immersed himself in the study of Grothendieck's anabelian geometry, formulated in 1984 in Esquisse d'un programme. His early work, jointly with G. Shabat, concerned Dessins d'enfants, the study of Galois groups of curves over number fields via their representation by special graphs on Riemann surfaces. The inspiration came partially from a result of Belyi, who proved that all such curves admit special meromorphic functions, with only three ramification points; moreover, the existence of such functions characterises these curves among all complex projective curves. At that time, it seemed that this result might open the door to the solution of major open problems in arithmetic geometry, such as Mordell's conjecture and Fermat's last theorem, as well as another important conjecture that is still open: the Section Conjecture of Grothendieck. Vladimir's interest in this area showed his determination, early on, to tackle the most difficult and challenging conjectures in mathematics.

His next project was a proof of the reconstruction of hyperbolic curves over a natural class of ground fields from their étale fundamental groups. This is the first step toward the Section Conjecture. His joint papers with M. Kapranov, on what seemed to be not very popular issues in category theory ( $n$-categories, $\infty$-groupoids and higher braid groups), turned out to be crucial for his work in algebraic geometry over the next two decades, as well as for univalent foundations. Already at that time, he was stating casually: "If the categorical framework works out, the Bloch-Kato conjecture will follow trivially."

His research interfered with his undergraduate work and he did not show up for classes or exams. After eventually quitting Moscow State University, he moved, in 1990, to Harvard University, where he became a $\mathrm{PhD}$ student. He graduated in 1992 and, after one year at the Institute for Advanced Study, he returned to Harvard as a Junior Fellow of the Harvard Society of Fellows.

All these years, he was relentlessly working on foundational problems; his meagre publication record between 1991 and 1995 is in stark contrast with the intensity of his investigations. Then came an avalanche of papers that radically changed algebraic geometry, settling major open conjectures (e.g. the construction of the derived category of motives, the Milnor conjecture and the more general Bloch-Kato conjecture) and introducing powerful new techniques. These conjectures postulated a deep and highly nontrivial connection between the geometry of algebraic varieties and their Galois symmetries. As Voevodsky's proof showed, this bridge required radically new concepts; no simplifications of his original proof have emerged despite intense efforts by geometers and algebraists.

Voevodsky's main achievement was the creation of an amalgam of homotopy theory and algebraic geometry. Both theories deal with objects of geometric origin but on the basis of completely different conceptions: while homotopy theory emphasises flexibility, algebraic geometry is rather rigid - algebraic varieties resist small, local perturbations. Mixing these essentially incompatible worlds in a meaningful context required a leap of faith and an enormous, prolonged effort.

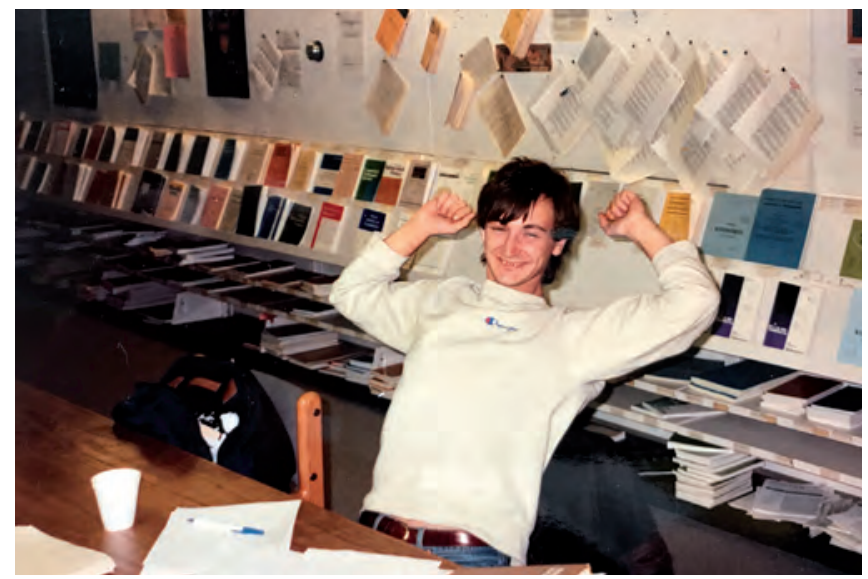

In the Harvard Mathematical Department Library (1993). 


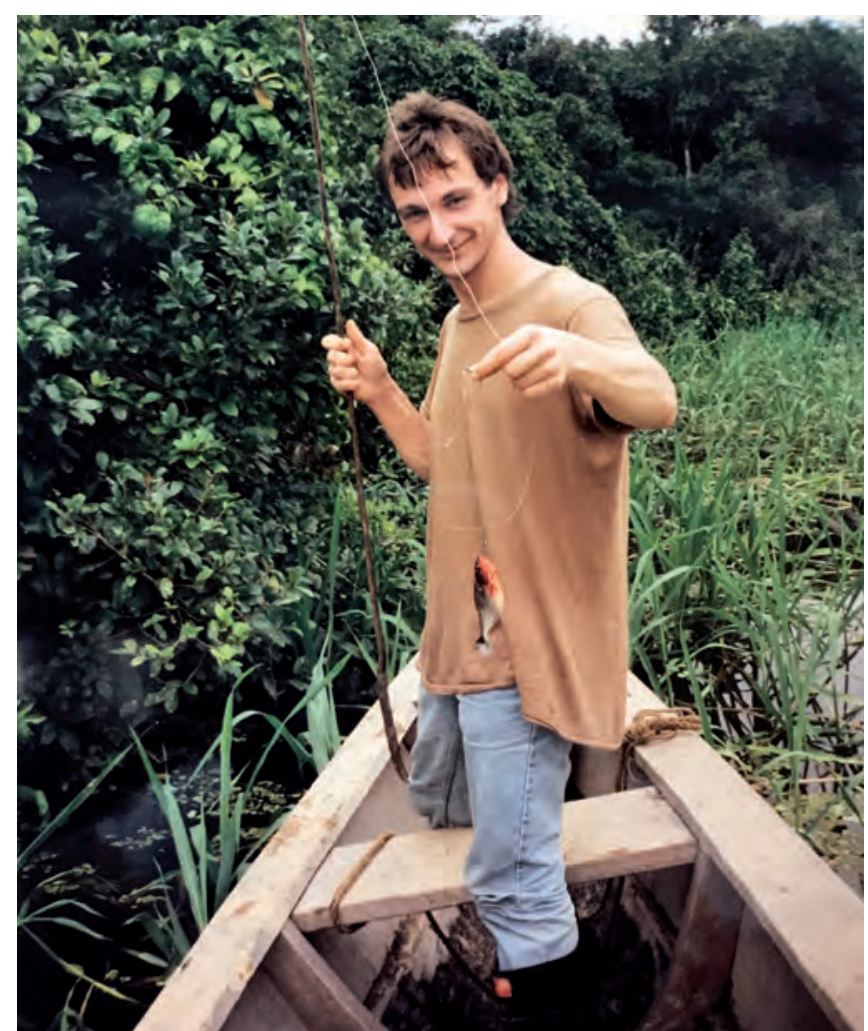

In the Amazon, with a piranha on the hook (1993).

After a short period of teaching at Northwestern University, Voevodsky moved to the Institute of Advanced Study. In his words, he began to "lose motivation" for work in algebraic geometry around 2003, having completed a vast research programme. He started taking computer science courses at Princeton University; private conversations with him frequently revolved around the nature of correctness, truth and proof in mathematics. This was triggered by the attempts and failures of several mathematicians working on big categorical structures that were too formidable for paper-and-pencil analysis. Voevodsky was led to a more general question of whether mathematicians had the right tools to explore difficult new areas like the highly complex theories he was interested in.

Voevodsky learned that (starting with N.G. de Bruijn) computer scientists and logicians had created automated proof assistants such as Mizar, Coq and Nuprl. However, to be applicable in practice, the mathematical proofs first had to be fully formalised in logical systems that the proof assistants could implement, e.g. set theory or other type theories. This step already presents a daunting obstacle in any minimally nontrivial situation. The analysis of existing proof assistants convinced Voevodsky that computers could, in principle, check mathematical proofs but that none of the available systems were up to this task on a fundamental rather than just a technical level.

With his usual vigour and tenacity, Voevodsky decided to create the foundations for this area at the interface of mathematics and computer science. His main insight was based on his previous experience in mathematics: the introduction of ideas of homotopy theory into the theory of types. His univalence axiom postulates that homotopy-equivalent objects share the same formal properties. Voevodsky was convinced that a systematic use of his univalent foundations would lead to the construction of practical proof assistants.

Again, there was a substantial gap in his publication record, followed by a burst of activity, starting in 2014, with $15(!)$ papers

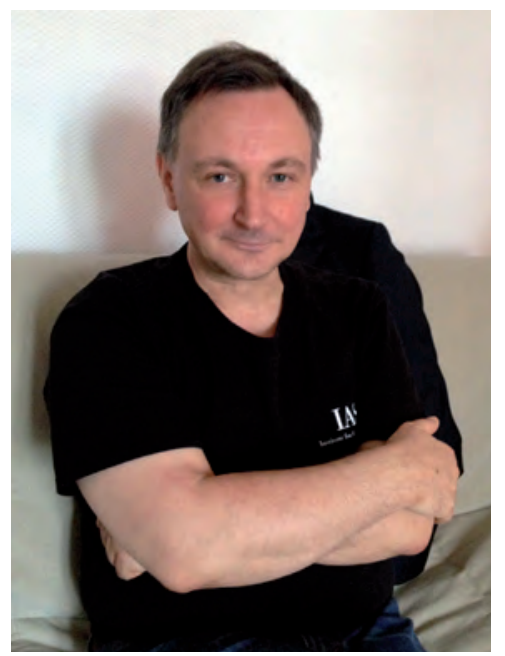

At the Institut Henri Poincaré, Paris (2014). posted on arXiv, three of them in June of this year alone. The introduction of univalence has already created great excitement in the community working with type theories in mathematics, philosophy and computer science. Initial prototype "univalent" proof assistants were created and Voevodsky and his collaborators embarked on a project to build a comprehensive library of mathematics, based on univalent foundations rather than set theory and rigorously checked by computer.

Voevodsky always said that this work was only a prototype and that the ultimate foundations for computerchecked mathematics had yet to be perfected. His vision sparked many ongoing research efforts, e.g. to find a constructive interpretation of univalence and to explore the use of univalence in various areas of mathematics.

His sudden and untimely death came as a shock to his colleagues and friends. He stands out as one of the giants of modern mathematics. The full impact of his ideas is still to be understood and appreciated.

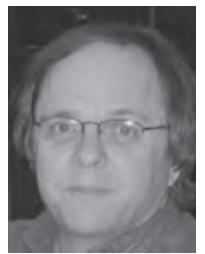

Dr. Mark Bickford (PhD 1983 in mathematics and logic from UW Madison) is currently a visiting scientist at Cornell University, computer science. His interests are in constructive mathematics and type theory. He is a user and developer of Nuprl and is formalizing the constructive model for Univalence in Nuprl.

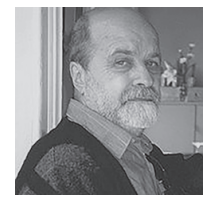

Fedor Bogomolov is Professor of Mathematics at the Courant Institute, NYU. He works in algebraic geometry.

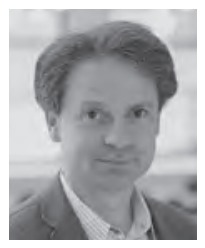

Yuri Tschinkel is Professor of Mathematics at the Courant Institute, NYU, and Director of Mathematics and the Physical Sciences at the Simons Foundation. He research interests are in algebraic geometry and number theory. 